\title{
SFOA: Sun Flower Optimization Algorithm to Solve Optimal Power Flow
}

\author{
Thomas Thangam \\ International Maritime College Oman, Sohar \\ Peripheral Rd, Liwa, Oman \\ thomasthangam13@gmail.com
}

\section{Hussein A. Kazem}

Sohar University

Al Jamiah Street Sohar OM, 311, Oman

\author{
Muthuvel K \\ Noorul Islam Centre for Higher Education \\ Kumarakovil, Tamil Nadu, India
}

\begin{abstract}
In electric power systems engineering, the set of optimization problems called cooperatively as OPF, which is the main sensibly significant as well as well-researched subfields of constrained nonlinear optimization. Moreover, OPF have the benefits of an affluent research history, novelty, and publication since its five decades ago. However, entry into OPF research is an intimidating job for the uninitiated-both because of the sheer volume of literature. In additionally, it is due to the OPF's ubiquity within the electric power systems community, which has led authors to presume an immense deal of prior knowledge that readers unknown with electric power systems may not possess. In this paper, a novel Sunflower Optimization Algorithm (SFOA) is proposed, which is enthused by the orientation of sunflower towards the sun to resolve constrained OPF problem. The proposed method is simulated to optimize the objective models namely fuel cost, voltage profile, emission, voltage stability, and active power loss. Moreover, the proposed method is compared with the conventional algorithms in the IEEE 30-bus, 57-bus, and 118-bus power systems. Finally, the simulation outcomes exhibit that the proposed method performs superior to other conventional approaches.
\end{abstract}

Keywords: Optimal Power Flow; Control Variables; Constraints; Sunflower; Optimization Algorithms

\begin{tabular}{ll} 
Nomenclature & \\
\hline Abbreviations & Descriptions \\
\hline OPF & Optimal Power Flow \\
LP & Linear Programming \\
MSA & Moth Swarm Algorithm \\
ANN & Artificial Neural Network \\
GWO & Grey Wolf Optimization \\
GSA & Gravitational Search Algorithm \\
PSTs & Phase-shifting transformers \\
FPA & Flower Pollination Algorithm \\
ADMM & Alternating Direction Multiplier Method \\
MILP & Mixed-Integer Linear Programming \\
PSO & Particle Swarm Optimization algorithm \\
VD & Voltage Deviations \\
APP & Auxiliary Problem Principle \\
GA & Genetic Algorithm \\
QP & Quadratic Programming \\
MFO & Moth Flame Optimization \\
WDF & Weibull Distribution Function \\
\hline
\end{tabular}

\section{Introduction}

Nowadays, electricity demand has been increasing day by day. On the basis of the thermal power plant, the production of energy meets $70 \%$ all over the world. Hence, the cost of fuel gets increases due to the huge demand for fuel [1]. At all load conditions, the economic operation is determined to contribute power from the well effectual plant. Based on the equality constraints like reactive and active power demand, the economic dispatch highly concerns while Optimal Power Flow represents the power system constraints such as the security and the load. Moreover, the OPF is considered as an operational 
planning tool that has the ability to reduce the objective model without breaching any associated constraints.

In power system operation and planning, at the present time, the OPF considered as crucial research [2]. In planning problems, OPF is extensively exploited or to locate optimal generation schedules in reactive and active power on an operational level, which reduce the operating system costs dependent on grid constraints [20].

OPF plays a significant problem to integrate electrical energy system with the wind energy generations. When processing $\mathrm{OPF}$, important issues such as operating and planning energy systems occur while it is integrated into remote areas. One of the main objectives of the operating power flow is to control the fundamental dispatching procedure, demands to distribute as well as to reduce the transmission loss. Furthermore, it increases and idea in minimizing the whole generation cost as well as the requirements and the operation procedure. For the users, the unavoidable space is developed by electrical energy, an industrialist who employs the energy system and all the stockholders in the practice of energy with the assist of wind power.

Earlier, numerous researchers examined on several optimization algorithms that exhibit the different issues and how it obtain and resolved with assist for the solution of the OPF [4]. Generally, two kinds of algorithms are exploited namely traditional and intelligent algorithms in order to resolve the OPF problems. The optimal power flow solutions methods in traditional methods like LP, QP, Newton Raphson, and Gradient methods are presented in the state of the art. One of the trendy methods, APP, was exploited to parallelize the problem in OPF solution [9]. The intelligent algorithms in order to solve the OPF solutions are such as PSO [12], GA [11], ANN [14], and GWO Algorithm [13]. In recent times, the ADMM has shown high interest [10]. These aforesaid algorithms engross the multiplier updating procedure that is frequently attained by a central coordinator [21].

The main contribution of this paper is to propose a novel Sunflower Optimization Algorithm (SFOA), this algorithm is exploited to solve the OPF in the proposed system. Moreover, the proposed method is simulated to optimize the objective functions namely fuel cost, voltage profile, emission, voltage stability, and active power loss.

This paper is organized as follows: Section 2 describes the literature review section and section 3 defines the problem formulation. Section 4 describes the proposed sun flower optimization algorithm for $\mathrm{OPF}$ problem. Section 5 summarizes the results and discussions sections and section 6 describes the conclusion of the paper.

\section{Literature Review}

In 2018, Shilaja C. and Arunprasath T [1], presented a hybrid MSA-GSA algorithm, was integrated by both the GSA and MSA for power systems with Wind energy sources. For showing the irregular nature of the wind farm, the WDF was exploited. In order to solve the objective models for failing the cost of fuel for the minimized power loss, with and without wind power test cases were considered. At last, the experimentation outcomes were examined on IEEE 30-bus, 57 bus and 118 bus without and with wind power.

In 2019, Zhangliang Shen et al [2], presented a novel technique in order to expand convex relaxations of OPF issues to comprise ZIP load models representations. In order to unwind the expressions of voltage magnitude, the geometric relationships approach was exploited with the help of the reference bus' phase angle knowledge. Hence convex demonstration of ZIP load models was enabled to form the constantcurrent mechanism. For the OPF problem, the presented technique was exploited for quadratic convex programming relaxations, second-order cone, semi-definite.

In 2019, P. Fortenbacher and T. Demiray [3], proposed a new technique to estimate the nonlinear AC OPF into tractable QP/LP. Moreover, for power system planning as well as operation the OPF problems was exploited. In the structure of absolute value functions, a linear branch and power flow estimate was considered. A power loss estimation, which was appropriate to wrap a broader operating range, was derived. Here, the main notations were to detain the losses of power and flows of power in the full decision variable domain.

In 2019, Guido Coletta et al [4], addressed a problem in weather-based method, which was identified as a major potential facilitating technique in order to increase the system suppleness using the components of real power loadability. Nevertheless, in real operation circumstances, the consumption of this method could be acutely cooperation because of the uncertainty data effects. The investigation of reliable approaches that concentrated at managing and representing uncertainties indicates the majority of pertinent issues to resolve. Equipped with this idea, correlated and multiple uncertainties presence, this article supports the function of Affine Arithmetic in consistent resolving OPF problems on the basis of weather. 
In 2018, Tao Ding et al [5], developed a PSTs technique that has the ability to regulate and reduce the total generation cost in OPF problems. By managing a small fraction of PSTs, multiple optimal solutions of PST angle modification and enhanced economy can attain under the perception. Therefore, a MILP model was presented in order to optimally decide the PSTs subset for angle modification. Finally, the simulation outcomes on numerous test systems such as large-scale systems demonstrate that the proposed method can offer enhanced economic.

In 2019, Zhifang Yang et al [6], presented a competent OPF method in order to hybridize the AC-DC grids with discrete control devices. In both $\mathrm{AC}$ and $\mathrm{DC}$ grids, a successive linear approximation technique for the power flow equations was developed. Moreover, for the converter station, the operational constraints were methodically examined. The convexity for the modeling of the VSC, the linearization of the power flow equations was presented. In order to hold the extremely nonlinear converter losses, an invented branch was augmented to the AC grid. Moreover, an iterative solving method was presented for the model of the OPF.

In 2019, Wentian Lu et al [7], developed a fully OPF decentralized method for multi-area interconnected power systems on the basis of the distributed interior-point technique. Moreover, the regional modification equations were converted into a parametric QP issue. In order to tackle the decentralized OPF problems, this technique was considered as a novel technique, as well as the convergence property of this technique was analyzed. In addition, the aforesaid decentralized technique likes the similar performance of the convergence as well as, the precision as same as the centralized interior-point technique.

In 2018, Wei Wei et al [8], worked on a novel class of OPF problems in the distribution systems. By modifying the demands, the elastic loads act in response with the real-time nodal prices. In order to identify equilibrium, a fixed point iteration method was recommended. To evaluate the convergence, a brief criterion was developed.

\section{Problem Formulation}

In general, an OPF is contemplated as a nonlinear and non-convex optimization issue. It is used to decrease objectives of a particular power system that subjects to numerous equality and inequality constraints. It is done by deciding the optimal control variables in order to present load settings. Eq. (1), (2), and (3) presents the objective model of OPF.

$$
\begin{aligned}
& \text { Min }: 1(\mathrm{y}, \mathrm{v}) \\
& \text { subject to }: \mathrm{m}(\mathrm{y}, \mathrm{v})=0 \\
& \mathrm{n}(\mathrm{y}, \mathrm{v}) \leq 0
\end{aligned}
$$

In eq. (1), y represents the system vector state or dependent variables, $\mathrm{v}$ represents the vector of independent or control variables, $\mathrm{l}(\mathrm{y}, \mathrm{v})$ represents the objective model to be reduced, $\mathrm{m}(\mathrm{y}, \mathrm{v})$ represents the equality constraints, and $\mathrm{n}(\mathrm{y}, \mathrm{v})$ indicates the inequality constraints. The state and the control variables of the OPF problem are listed below.

\section{a) Control variables:}

As eq. (4), the set of parameters that controlled by the power flow equations, are indicated with respect to the decision vector.

$$
\mathrm{v}=\left[\mathrm{M}_{\mathrm{h}_{2}}, \ldots . ., \mathrm{M}_{\mathrm{F}_{\mathrm{NF}}}, \mathrm{U}_{\mathrm{f}_{1}}, \ldots . . \mathrm{U}_{\mathrm{f}_{\mathrm{nf}}}, \mathrm{TR}_{1},,,,, \mathrm{TR}_{\mathrm{NT}}, \mathrm{N}_{\mathrm{C}_{1}}, \ldots, \mathrm{N}_{\mathrm{C}_{\mathrm{Nc}}}\right\rfloor
$$

In eq. (4), $\mathrm{M}_{\mathrm{h}}$ denotes the active power generator, $T R$ is the transformer tap, $\mathrm{U}_{\mathrm{f}}$ denotes the voltage magnitude generators, $\mathrm{N}_{\mathrm{C}}$ denotes the shunt reactive power VAR compensators, $\mathrm{N}_{\mathrm{T}}$ represents the amount of regulating transformers and $\mathrm{N}_{\mathrm{c}}$ represents the shunt VAR compensators units, correspondingly.

\section{b) State variables}

The eq. (5) indicates the set of variables that denote the power system.

$$
\mathrm{y}=\left[\mathrm{M}_{\mathrm{h}_{1}}, \mathrm{U}_{\mathrm{f}_{1}}, \ldots . ., \mathrm{M}_{\mathrm{F}_{\mathrm{NF}}}, \mathrm{N}_{\mathrm{h}_{1}}, \ldots ., \mathrm{N}_{\mathrm{F}_{\mathrm{Nh}}}, \mathrm{R}_{\mathrm{f}_{\mathrm{f}}}, \ldots . \mathrm{R}_{\mathrm{f}_{\mathrm{nf}}}\right]
$$

In eq. (5), $\mathrm{M}_{\mathrm{h}_{1}}$ represents the generation of active power at slack-bus, $\mathrm{U}_{\mathrm{f}}$ denotes the voltage magnitude at load bus, $\mathrm{N}_{\mathrm{h}}$ represents the reactive power outputs of the generators, and $R$ represents the apparent power flow, correspondingly. $\mathrm{N}_{\mathrm{F}}$ represents the number of load buses $(\mathrm{M}-\mathrm{N}), \mathrm{N}_{\mathrm{h}}$ represents the generators buses $(\mathrm{M}-\mathrm{U})$, and $\mathrm{N}_{\mathrm{nl}}$ represents the transmission lines, correspondingly. 


\subsection{Constraints}

The OPF problem needs to complete both inequality as well as equality constraints. As equality constraint, the power balance constraints are represented. The power system operating limits components are represented as inequality constraints.

\section{a) Equality constraints}

As eq. (6) and (7), the balance of the reactive and active power is exploited, and these constraints indicate the typical load flow equations.

$$
\begin{aligned}
& \left.\mathrm{M}_{\mathrm{hi}}-\mathrm{M}_{\mathrm{di}}-\mathrm{U}_{\mathrm{i}} \sum_{\mathrm{j}=1}^{\mathrm{na}} \mathrm{U}_{\mathrm{j}}\left[\mathrm{H}_{\mathrm{ij}} \cos \Delta_{\mathrm{i}}-\Delta_{\mathrm{j}}\right)+\mathrm{A}_{\mathrm{ij}} \sin \left(\Delta_{\mathrm{i}}-\Delta_{\mathrm{j}}\right)\right]=0 \forall i \varepsilon n a \\
& \mathrm{~N}_{\mathrm{hi}}-\mathrm{N}_{\mathrm{di}}-\mathrm{U}_{\mathrm{i}} \sum_{\mathrm{j}=1}^{\mathrm{na}} \mathrm{U}_{\mathrm{j}}\left[\mathrm{H}_{\mathrm{ij}} \sin \left(\Delta_{\mathrm{i}}-\Delta_{\mathrm{j}}\right)-\mathrm{A}_{\mathrm{ij}} \cos \left(\Delta_{\mathrm{i}}-\Delta_{\mathrm{j}}\right)\right]=0 \forall \mathrm{i} \varepsilon n a
\end{aligned}
$$

Where, $\mathrm{M}_{\mathrm{d}}$ indicates the demand of active load, $\mathrm{N}_{\mathrm{d}}$ represents the demand of reactive load, na indicates the total number of buses, $\mathrm{N}_{\mathrm{h}}$ indicates the reactive power generator, $\mathrm{H}_{\mathrm{ij}}$ indicates the transfer conductance and $\mathrm{A}_{\mathrm{ij}}$ represents the and susceptance among bus $\mathrm{i}$ and bus $\mathrm{j}$, correspondingly. During the load flow procedure, these constraints are severely imposed that promises, which the optimal searched solution is possible.

\section{b) Inequality constraints:}

These constraints indicate the operation of power system limits is listed below.

(i) Constraints of generation:

In eq. (8), (9) and (10), using the upper and lower limits the real power, the voltages, and reactive power of the generators are limited for stable operation.

$$
\begin{aligned}
& \mathrm{M}_{\mathrm{hi}}^{\min } \leq \mathrm{M}_{\mathrm{hi}} \leq \mathrm{M}_{\mathrm{hi}}^{\max } 0 \forall \mathrm{i \varepsilon NH} \\
& \mathrm{N}_{\mathrm{hi}}^{\min } \leq \mathrm{N}_{\mathrm{hi}} \leq \mathrm{N}_{\mathrm{hi}}^{\max } 0 \forall \mathrm{i} \varepsilon \mathrm{NH} \\
& \mathrm{U}_{\mathrm{hi}}^{\min } \leq \mathrm{U}_{\mathrm{hi}} \leq \mathrm{U}_{\mathrm{hi}}^{\max } 0 \forall \mathrm{i} \varepsilon \mathrm{NH}
\end{aligned}
$$

(ii) Constraints of Transformer

Eq. (11) states the transformers tap settings should be limited by their upper and lower limits.

$$
\mathrm{TR}_{\mathrm{i}}^{\min } \leq \mathrm{TR}_{\mathrm{i}} \leq \mathrm{TR}_{\mathrm{i}}^{\max } 0 \forall i \varepsilon \mathrm{NTR}
$$

(iii) Security constraints:

The constraints of transmission line loadings and load buses voltage magnitudes have to be limited within their limits.

(iv) Constraints of Shunt VAR compensator

$$
\begin{aligned}
& \mathrm{U}_{\mathrm{Ai}}^{\min } \leq \mathrm{U}_{\mathrm{Ai}} \leq \mathrm{U}_{\mathrm{Ai}}^{\max } 0 \forall \mathrm{i} \varepsilon \mathrm{NF} \\
& \mathrm{R}_{\mathrm{fi}} \leq \mathrm{U}_{\mathrm{Ai}}^{\max } 0 \forall \mathrm{i} \varepsilon \mathrm{l}
\end{aligned}
$$

Eq. (14), the shunt VAR compensators are limited by their limits.

$$
\mathrm{N}_{\mathrm{ci}}^{\min } \leq \mathrm{N}_{\mathrm{ci}} \leq \mathrm{N}_{\mathrm{ci}}^{\max } 0 \forall \mathrm{i \varepsilon NC}
$$

\subsection{Handling of Constraints}

The inequality constraints of dependent variables comprise magnitude of load bus voltage; generation output of real power at the slack bus, generation of reactive power output as well as line loading are integrated into the comprehensive objective model to maintain the dependent variables within their allowable limits and to refuse any impossible solution.

In eq. (15) represents the penalty function, which is described using quadratic terms [15].

$$
\text { Penality }=\mathrm{O}_{\mathrm{M}}\left(\mathrm{M}_{\mathrm{h} 1}-\mathrm{M}_{\mathrm{hl}}^{\lim }\right)^{2}+\mathrm{O}_{\mathrm{N}} \sum_{\mathrm{i}=1}^{\mathrm{Nh}}\left(\mathrm{N}_{\mathrm{hi}}-\mathrm{N}_{\mathrm{hi}} \lim \right)^{2}+\mathrm{O}_{\mathrm{U}} \sum_{\mathrm{i}=1}^{\mathrm{Nf}}\left(\mathrm{U}_{\mathrm{Ai}}-\mathrm{U}_{\mathrm{Ai}} \lim ^{2}+\mathrm{O}_{\mathrm{R}} \sum_{\mathrm{i}=1}^{\mathrm{nf}}\left(\mathrm{R}_{\mathrm{fi}}-\mathrm{R}_{\mathrm{fi}} \lim \right)^{2}\right.
$$

In eq. (15), $\mathrm{O}_{\mathrm{M}}, \mathrm{O}_{\mathrm{N}}, \mathrm{O}_{\mathrm{U}}$ and $\mathrm{O}_{\mathrm{R}}$ represents the factors of penalty that possess a high positive value that is indicated as 100 excluding the load voltage (KV) is set to 100,000 . In eq. (16), $\mathrm{y}^{\text {lim }}$ represents the debased value of the limit dependent variable $y$.

$$
y^{\lim }=\left\{\begin{array}{l}
y^{\max } \text { if } y>y^{\max } \\
y^{\min } \text { if } y>y^{\min }
\end{array}\right.
$$




\section{Proposed Sun Flower Optimization Algorithm (SFOA)}

In this section, the detailed procedure of the proposed Sun Flower Optimization Algorithm (SFOA) [16] is discussed in order to solve the OPF problem.

\section{(a) Basic Concepts:}

Each and every day, the sunflower cycle remains the same, the sunflower rouse and follows the sun similar to the needles of a clock. During the night, the sunflower explores the opposing way to wait over again for their exodus next morning. In [17], a novel technique was presented on the basis of the flower pollination procedure of flowering plants taking into consideration of the biological procedure of reproduction.

\section{(b) Mathematical Description of proposed algorithm:}

In the proposed algorithm, the uncharacteristic sunflowers behavior in the search for the optimal point of reference in the direction of the sun has considered. Moreover, in a random manner, the pollination is contemplated besides the least distance among the flower $i$ and the flower $\mathrm{i}+1$. Each patch of the flower frequently liberates millions of pollen gametes in fact. On the other hand, presume that each sunflower merely generates one pollen gamete as well as reproduce independently for ease.

The inverse square law radiation is represented as an additional significant nature-based optimization. Here, it exhibits the radiation intensity, which is inversely proportional to the square of the distance. In proportion, the radiation intensity minimizes and so the square of the distance gets increases. If the distance gets thrice the intensity minimizes to a factor 9 , and if the distance gets double, the intensity minimizes to a factor 4 , and so on. In this scenario, the quantity of radiation obtained will be higher when the distance from the plant to the sun is lesser, as well as it will be inclined to steady in this surrounding area. Conversely, if the distance from the plant to the sun is higher, the quantity of heat obtained by it will be lesser. In the proposed technique, the similar steps are followed that may acquire more steps to obtain as near as probable to the global optimum (sun) [18]. After that, the quantity of heat $H$ obtained by each plant is stated in eq. (17). Here, $S$ indicates the source power and $\mathrm{d}_{\mathrm{i}}^{2}$ represents the distance among the current optimal and the plant $i$.

$$
\mathrm{H}_{\mathrm{i}}=\frac{\mathrm{S}}{4 \pi \mathrm{d}_{\mathrm{i}}^{2}}
$$

The sunflowers direction to the sun is represented in eq. (18). Eq. (19) is exploited to compute the step of the sunflowers on the direction $m$.

$$
\begin{aligned}
\vec{r}_{i} & =\frac{\mathrm{Y}^{*}-\mathrm{Y}_{\mathrm{i}}}{\left\|\mathrm{Y}^{*}-\mathrm{Y}\right\|}, \mathrm{i}=1,2, \ldots, \mathrm{n}_{\mathrm{s}} \\
\mathrm{m}_{\mathrm{i}} & =\lambda \times \mathrm{S}_{\mathrm{i}}\left(\left\|\mathrm{Y}_{\mathrm{i}}+\mathrm{Y}_{\mathrm{i}-1}\right\|\right) \times\left\|\mathrm{Y}_{\mathrm{i}}+\mathrm{Y}_{\mathrm{i}-1}\right\|
\end{aligned}
$$

In eq. (19), $\lambda$ indicates the constant value, which states a "inertial" displacement of the plants, $\mathrm{S}_{\mathrm{i}}\left(\left\|\mathrm{Y}_{\mathrm{i}}+\mathrm{Y}_{\mathrm{i}-1}\right\|\right)$ indicates the pollination probability, that is the sunflower $\mathrm{i}$ pollinates with its adjacent neighbor i-1 producing a new individual in an arbitrary location, which deviates consistent with each distance among the flowers i.e., individuals nearer to the sun may acquire lesser steps for a search of local modification when distant is more individuals will move usually. Also, it is essential to limit the utmost step specified by each individual, in turn not to leave out areas flat to be global least candidates. The utmost step can be defined as eq. (20), whereas $Y_{\max }$ represents upper bounds, $Y_{\text {min }}$ represents lower bounds, $\mathrm{N}_{\text {pop }}$ represents a number of plants in the total population.

$$
\mathrm{m}_{\text {max }}=\left\|\frac{\mathrm{Y}_{\text {max }}-\mathrm{Y}_{\min }}{2 \times \mathrm{N}_{\text {pop }}}\right\|
$$

The updating formula of the proposed method is represented in eq. (21).

$$
\overrightarrow{\mathrm{Y}}_{\mathrm{i}+1}=\overrightarrow{\mathrm{Y}}_{\mathrm{i}}+\mathrm{m}_{\mathrm{i}} \times \overrightarrow{\mathrm{r}}_{\mathrm{i}}
$$




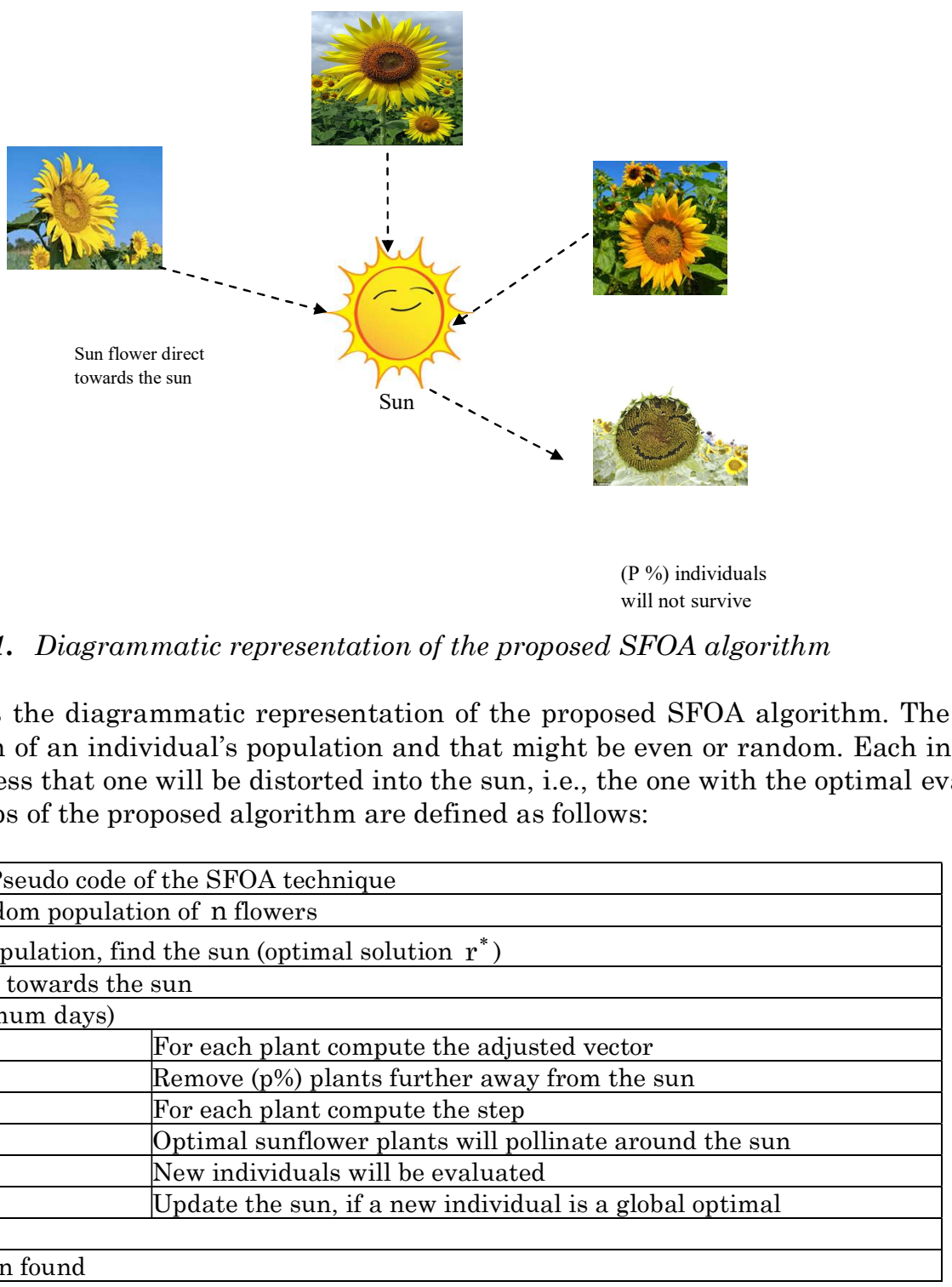

end while

\begin{tabular}{|c|c|}
\hline $\begin{array}{l}\text { Algorithm 1: Pseudo ce } \\
\text { Initialize a random pop }\end{array}$ & $\begin{array}{l}\text { of the SFOA technique } \\
\text { ion of } \mathrm{n} \text { flowers }\end{array}$ \\
\hline In the initial population & d the sun (optimal solution $r^{*}$ ) \\
\hline Adjust all plant toward & sun \\
\hline while $(1<$ Maximum day & \\
\hline & For each plant compute the adjusted vector \\
\hline & Remove (p\%) plants further away from the sun \\
\hline & For each plant compute the step \\
\hline & Optimal sunflower plants will pollinate around the sun \\
\hline & New individuals will be evaluated \\
\hline & Update the sun, if a new individual is a global optimal \\
\hline end while & \\
\hline Optimal solution found & \\
\hline
\end{tabular}

\section{Results and Discussions}

\subsection{Simulation Procedure}

In this section, the simulation experiment of six different scenarios was done, and it was tested in the IEEE 30-bus, 57-bus, and the 118-bus systems. Here, the SFOA method was exploited as the proposed method and it was compared with the conventional algorithms such as FPA [17], PSO [12] and MFO [19].

Here, 6 different scenarios were considered to examine the complex system for both the single and multi-objective power flow. First three scenarios such as total fuel cost, emission, and active power loss were considered for single-objective model and remaining three such as fuel cost and the transmission power loss, the fuel cost and VD and L-index were considered for multi-objective power flow.

The objective model taking into consideration for the minimization of the total fuel cost of power generation is represented as the first single objective model, and it stated in eq. (22), where $\mathrm{x}_{\mathrm{i}}, \mathrm{y}_{\mathrm{i}}$ and $z_{i}$ represents the coefficient cost of $i^{\text {th }}$ generator.

$$
\mathrm{f}_{\mathrm{c}}=\left(\sum_{\mathrm{i}=1}^{\mathrm{NH}} \mathrm{x}_{\mathrm{i}} \mathrm{M}_{\mathrm{hi}}^{2}+\mathrm{y}_{\mathrm{i}} \mathrm{M}_{\mathrm{hi}}^{2}+\mathrm{z}_{\mathrm{i}}\right)+\operatorname{Penality}(\$ / \mathrm{h})
$$


The objective model in order to reduce the emission level of the two significant pollution gases such as $\mathrm{SO}_{\mathrm{x}}$ and $\mathrm{NO}_{\mathrm{x}}$ for the single objective model that can be computed as using eq. (23), where $\alpha_{\mathrm{i}}, \beta_{\mathrm{i}}, \delta_{\mathrm{i}}$ and $\lambda_{i}$ represents the coefficient cost of $i^{\text {th }}$ generator

$$
\mathrm{f}_{\mathrm{e}}=\left(\sum_{\mathrm{i}=1}^{\mathrm{NH}} \alpha_{\mathrm{i}} \mathrm{M}_{\mathrm{hi}}^{2}+\beta_{\mathrm{i}} \mathrm{M}_{\mathrm{hi}}^{2}+\delta_{\mathrm{i}}+\phi_{\mathrm{i}} \mathrm{e}^{\left(\lambda_{\mathrm{i}} \mathrm{M}_{\mathrm{hi}}\right)}\right)+\text { Penality }(\text { ton } / \mathrm{h})
$$

For a single objective model, the minimization of the active power loss for each transmission line is stated in eq. (24).

$$
\mathrm{f}_{\mathrm{p}}=\sum_{\mathrm{i}=1}^{\mathrm{nf}} \sum_{\mathrm{i}=1}^{\mathrm{nf}} \mathrm{H}_{\mathrm{ij}} \mathrm{U}_{\mathrm{i}}^{2}+\mathrm{U}_{\mathrm{i}}^{2}-2 \mathrm{U}_{\mathrm{i}} \mathrm{U}_{\mathrm{j}} \cos \left(\Delta_{\mathrm{i}}-\Delta_{\mathrm{j}}\right)+\text { Penalit( }(\mathrm{MW})
$$

The objective model to the minimization of the fuel cost and the transmission power loss for the multiobjective problem is stated in eq. (25).

$$
\begin{aligned}
\mathrm{f}= & \left(\sum_{\mathrm{i}=1}^{\mathrm{NH}} \mathrm{x}_{\mathrm{i}} \mathrm{M}_{\mathrm{hi}}^{2}+\mathrm{y}_{\mathrm{i}} \mathrm{M}_{\mathrm{hi}}^{2}+\mathrm{z}_{\mathrm{i}}\right)+\lambda_{\mathrm{m}} \sum_{\mathrm{i}=1}^{\mathrm{nf}} \sum_{\mathrm{i}=1}^{\mathrm{nf}} \mathrm{M}_{\mathrm{ij}} \mathrm{U}_{\mathrm{i}}^{2}+\mathrm{U}_{\mathrm{i}}^{2} \\
& -2 \mathrm{U}_{\mathrm{i}} \mathrm{U}_{\mathrm{j}} \cos \left(\Delta_{\mathrm{i}}-\Delta_{\mathrm{j}}\right)+\text { Penality }
\end{aligned}
$$

The multiple objective models to minimize the fuel cost and VD are summarized in eq. (26).

$$
\mathrm{f}=\left(\sum_{\mathrm{i}=1}^{\mathrm{NH}} \mathrm{x}_{\mathrm{i}} \mathrm{M}_{\mathrm{hi}}^{2}+\mathrm{y}_{\mathrm{i}} \mathrm{M}_{\mathrm{hi}}^{2}+\mathrm{z}_{\mathrm{i}}\right)+\lambda_{\mathrm{vd}} \sum_{\mathrm{i}=1}^{\mathrm{nf}}\left|\mathrm{U}_{\mathrm{F}_{\mathrm{i}}}-1\right|+\text { Penality }
$$

The minimization of fuel cost with voltage stability indicator named L-index, which is an important objective model for power system operation and planning, summarized in eq. (27).

$$
\mathrm{LI}_{\mathrm{i}}=\left|1-\sum_{\mathrm{i}=1}^{\mathrm{NH}} \mathrm{F}_{\mathrm{ji}} \frac{\mathrm{U}_{\mathrm{i}}}{\mathrm{U}_{\mathrm{j}}}\right| \forall \mathrm{j}=1,2, \ldots \ldots, \mathrm{NF}
$$

\subsection{IEEE 30 Bus System}

This section summarizes the efficiency of the proposed method in the IEEE 30 bus system. Table 1 shows the efficacy of the proposed technique for both the single and multi-objective models. Here, the performance of the proposed technique shows better result than the conventional techniques. Table 2 shows the computation time of the proposed method and conventional techniques. Here, the proposed technique is $0.6 \%$ better than the FPA method, $0.82 \%$ better than the PSO method, and $0.54 \%$ better than the MFO method.

Table 1. Performance analysis of the proposed and existing methods on IEEE 30 bus system

\begin{tabular}{lllll}
\hline Objective model & FPA & PSO & MFO & SFOA \\
\hline Fuel cost (\$/h) & 723.45 & 745.67 & 763.78 & 711.334 \\
Ploss(MW) & 4.545 & 4.346 & 4.654 & 4.341 \\
Emission(ton/h) & 0.456 & 0.445 & 0.448 & 0.399 \\
Qloss(MW) & 12.345 & 13.125 & 13.234 & 11.126 \\
VD(p.u) & 1.278 & 1.345 & 1.343 & 1.121 \\
L-index & 0.346 & 0.376 & 0.368 & 0.332 \\
\hline
\end{tabular}

Table 2. Computation time of the proposed and conventional algorithms on IEEE 30 bus system

\begin{tabular}{ll}
\hline Methods & Time (s) \\
\hline FPA & 14.79 \\
PSO & 15.34 \\
MFO & 14.33 \\
SFOA & 13.89 \\
\hline
\end{tabular}

\subsection{IEEE 57 Bus System}

This section demonstrates the performance analysis of the proposed and conventional methods on IEEE 57 Bus system. In Table 3, the objective models such as fuel cost, emission, Ploss, Qloss, VD, and L-index is exploited. The overall analysis exhibits the proposed technique is superior to the conventional techniques. Table 4 shows the computation time of both the proposed and conventional techniques. Here, the proposed technique is 10\% superior to FPA, 11\% superior to PSO, and 11.23\% superior to MFO. 
Table 3. Performance analysis of the proposed and conventional algorithms on IEEE 57 bus system

\begin{tabular}{lllll}
\hline Objective model & FPA & PSO & MFO & SFOA \\
\hline Fuel cost (\$/h) & 512.12 & 532.45 & 547.23 & 503.124 \\
Emission(ton/h) & 0.163 & 0.125 & 0.167 & 0.111 \\
Ploss(MW) & 3.236 & 3.153 & 3.248 & 3.043 \\
Qloss(MW) & 13.267 & 12.674 & 14.128 & 12.435 \\
VD(p.u) & 2.345 & 2.432 & 2.536 & 2.225 \\
L-index & 0.143 & 0.236 & 0.278 & 0.124 \\
\hline
\end{tabular}

Table 4. Computational time of the proposed and Existing methods on IEEE 57 bus system

\begin{tabular}{ll}
\hline Methods & Time (s) \\
\hline FPA & 16.12 \\
PSO & 16.90 \\
MFO & 15.12 \\
SFOA & 14.37 \\
\hline
\end{tabular}

\subsection{IEEE 118 Bus System}

In this section, the effectiveness of the proposed method in IEEE 118 bus system has been demonstrated. Table 5 summarizes the efficiency of the proposed method for both the single and multi-objective models. Here, the performance of the proposed method shows enhanced result than the conventional methods. Table 6 shows the computation time of the proposed method and conventional methods. Here, the proposed method is $22 \%$ better than the FPA method, $21 \%$ better than the PSO method, and $18 \%$ better than the MFO method.

Table 5. Performance analysis of the proposed and conventional algorithms on IEEE 118 bus system

\begin{tabular}{lllll}
\hline Objective model & FPA & PSO & MFO & SFOA \\
\hline Fuel cost (\$/h) & 849.69 & 876.37 & 884.32 & 823.43 \\
Ploss $(M W)$ & 5.435 & 5.426 & 5.267 & 5.176 \\
Emission(ton/h) & 0.284 & 0.256 & 0.278 & 0.232 \\
Qloss(MW) & 22.324 & 22.126 & 21.672 & 20.324 \\
VD(p.u) & 3.256 & 3.237 & 3.267 & 3.154 \\
L-index & 0.732 & 0.716 & 0.745 & 0.705 \\
\hline
\end{tabular}

Table 6. Computational time of the proposed and existing methods on IEEE 118 bus system

\begin{tabular}{ll}
\hline Methods & Time (s) \\
\hline FPA & 17.32 \\
PSO & 17.89 \\
MFO & 16.26 \\
SFOA & 13.43 \\
\hline
\end{tabular}

\section{Conclusion}

This paper presents a novel SFOA method in order to resolve the objective models of OPF in the power system. The proposed method was compared with the conventional methods such as FPA, PSO, and MFA. It was clear that the proposed method was appropriate in order to solve the complex problems and non- smooth problems from the attained results. Finally, the comparative analysis of the proposed technique and conventional techniques proves the superiority of the proposed concept. Moreover, the proposed method is $22 \%$ better than the FPA method, $21 \%$ better than the PSO method, and $18 \%$ better than the MFO method, which shows the proposed method is possible to discover suitable and precise solutions particularly for the multi-objective optimization issues and large-scale power systems.

\section{Compliance with Ethical Standards}

Conflicts of interest: Authors declared that they have no conflict of interest.

Human participants: The conducted research follows the ethical standards and the authors ensured that they have not conducted any studies with human participants or animals. 


\section{References}

[1] Shilaja C., Arunprasath T,"Optimal power flow using Moth Swarm Algorithm with Gravitational Search Algorithm considering wind power", Future Generation Computer Systems, vol. 98, pp. 708-715, September 2019.

[2] Zhangliang Shen, Zhinong Wei, Guoqiang Sun, Sheng Chen,"Representing ZIP loads in convex relaxations of optimal power flow problems", International Journal of Electrical Power \& Energy Systems, vol. 110, pp. 372385, September 2019.

[3] P. Fortenbacher, T. Demiray,"Linear/quadratic programming-based optimal power flow using linear power flow and absolute loss approximations",International Journal of Electrical Power \& Energy Systems, vol 107, pp. 680689, May 2019.

[4] Guido Coletta, Alfredo Vaccaro, Domenico Villacci, Duo Fang, Sasa Z. Djokic,"Affine arithmetic for efficient and reliable resolution of weather-based uncertainties in optimal power flow problems",International Journal of Electrical Power \& Energy Systems, vol. 110, pp. 713-724, September 2019.

[5] T. Ding, R. Bo, Z. Bie and X. Wang, "Optimal Selection of Phase Shifting Transformer Adjustment in Optimal Power Flow," IEEE Transactions on Power Systems, vol. 32, no. 3, pp. 2464-2465, May 2017.

[6] Z. Yang, H. Zhong, A. Bose, Q. Xia and C. Kang, "Optimal Power Flow in AC-DC Grids With Discrete Control Devices," IEEE Transactions on Power Systems, vol. 33, no. 2, pp. 1461-1472, March 2018.

[7] W. Lu, M. Liu, S. Lin and L. Li, "Fully Decentralized Optimal Power Flow of Multi-Area Interconnected Power Systems Based on Distributed Interior Point Method,"IEEE Transactions on Power Systems, vol. 33, no. 1, pp. 901-910, Jan. 2018.

[8] W. Wei, J. Wang and L. Wu, "Distribution Optimal Power Flow With Real-Time Price Elasticity," IEEE Transactions on Power Systems, vol. 33, no. 1, pp. 1097-1098, Jan. 2018.

[9] B. H. Kim, R. Baldick, "Coarse-grained distributed optimal power flow," IEEE Trans. Power Syst., vol. 12, no. 2, pp. 932-939, May 1997.

[10] E. Dall'Anese, H. Zhu, G. B. Giannakis, "Distributed optimal power flow for smart microgrids," IEEE Trans. Smart Grid, vol.4, no. 3, pp. 1464-1475, Sept. 2013.

[11] RT Satish, KT Reddy, "A technique to find optimal location for wavelet-based image watermarking using genetic algorithm", Machine Graphics \& Vision International Journal,vol. 20 (2), pp.173-196, 2011.

[12] SB Vinay Kumar, PV Rao, Manoj Kumar Singh,"Multi-culture diversity based self adaptive particle swarm optimization for optimal floorplanning",Multiagent and Grid Systems, vol14, no.1, pp.31-65, 2018.

[13] Singh, Gurinderpal \& Jain, V.K. \& Singh, Amanpreet. (2018). A prediction model for the smart Industries, aiding photovoltaic integrated greenhouse system in biogas heating. Computers \& Electrical Engineering.

[14] G Singh, VK Jain, A Singh, "Adaptive network architecture and firefly algorithm for biogas heating model aided by photovoltaic thermal greenhouse system",Energy \& Environment, vol. 29 (7), pp.1073-1097, 2018.

[15] B. Mahdad, K. Srairi, Blackout risk prevention in a smart grid based flexibleoptimal strategy using Grey Wolfpattern search algorithms, Energy Convers.Manage. 98 (2015) 411-429.

[16] Gomes, G. F., da Cunha, S. S., \& Ancelotti, A. C. A sunflower optimization (SFO) algorithm applied to damage identification on laminated composite plates. Engineering with Computers, p. 1-8, 2018.

[17] Yang X-S (2012) Flower pollination algorithm for global optimization. In: International conference on unconventional computing and natural computation. Springer, Berlin, pp 240-249.

[18] McInnes CR," Solar radiation pressure", Solar sailing", Springer, London, pp 32-55, 1999.

[19] C. Li, Z. Niu, Z. Song, B. Li, J. Fan and P. X. Liu, "A Double Evolutionary Learning Moth-Flame Optimization for Real-Parameter Global Optimization Problems," IEEE Access, vol. 6, pp. 76700-76727, 2018.

[20] Manisha Hooda,Dr. Y.K. Awasthi,Niharika Thakur and Dr. Anwar Shahzad Siddiqui,"A Hybrid CS-CSA Optimization Algorithm for Solving Optimal Power Flow in Single Objective Optimization",Journal of Computational Mechanics, Power System and Control (JCMPS), Volume 2, Issue 2, April 2019.

[21] SK.Mahammad Shareef and Dr.R.Srinivasa Rao,"A Hybrid Learning Algorithm for Optimal Reactive Power Dispatch under Unbalanced Conditions",Journal of Computational Mechanics, Power System and Control (JCMPS), Volume 1, Issue 1, October 2018. 